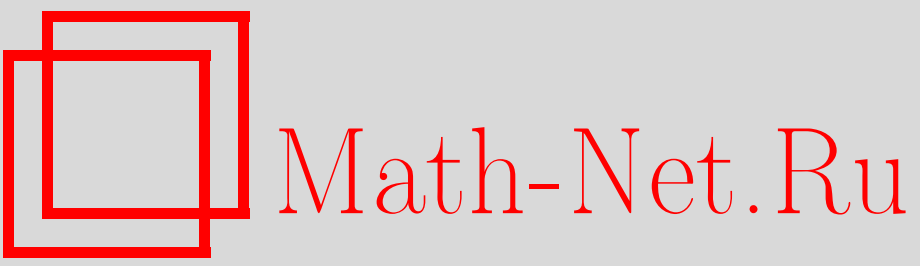

С. С. Мамонов, И. В. Ионова, А. О. Харламова, Скрытая синхронизация в системах фазовой автоподстройки частоты, Итоги науки и техн. Сер. Соврем. мат. и ее прил. Темат. обз., 2019, том 168, 71-79

DOI: https://doi.org/10.36535/0233-6723-2019-168-71-79

Использование Общероссийского математического портала Math-Net.Ru подразумевает, что вы прочитали и согласны с пользовательским соглашением

http://www.mathnet.ru/rus/agreement

Параметры загрузки:

IP : 35.173 .219 .149

26 апреля 2023 г., $17: 48: 48$ 


\title{
СКРЫТАЯ СИНХРОНИЗАЦИЯ В СИСТЕМАХ ФАЗОВОЙ АВТОПОДСТРОЙКИ ЧАСТОТЫ
}

\author{
(c) 2019 г. $\quad$ С. С. МАМОНОВ, И. В. ИОНОВА, А. О. ХАРЛАМОВА
}

\begin{abstract}
АннотАция. В работе рассматривается одна из разновидностей радиотехнических систем, а именно, система частотно-фазовой автоподстройки частоты (ЧФАПЧ). Математическая модель такой системы описывается системой дифференциальных уравнений с цилиндрическим фазовым пространством. Для системы ЧФАПЧ определены условия формирования скрытой синхронизации.
\end{abstract}

Ключевъе слова: скрытая синхронизация, система частотно-фазовой автоподстройки частоты, предельный цикл первого рода, квазисинхронный режим, режим биений, положительно инвариантное множество, вращение векторного поля.

\section{HIDDEN SYNCHRONIZATION IN PHASE LOCKED LOOP SYSTEMS}

\author{
(C) 2019 S. S. MAMONOV, I. V. IONOVA, A. O. KHARLAMOVA
}

\begin{abstract}
This paper is devoted to the analysis of frequency-phase locked loop systems (FPLL). The mathematical model of this system is a system of differential equations with a cylindrical phase space. For the FPLL system, we obtain conditions of latent synchronization.

Keywords and phrases: hidden synchronization, frequency-phase locked loop, limit cycle of the first kind, quasisynchronous mode, beat mode, positively invariant set, rotation of a vector field.
\end{abstract}

AMS Subject Classification: 34K05, 34K13, 34K60

Рассмотрим систему частотно-фазовой автоподстройки частоты (ЧФАПЧ), описываемую операторным уравнением (см. $[2,3,15,16])$

$$
p \sigma(t)+\Omega_{1} K_{1}(p) F_{1}(\sigma(t))+\Omega_{2} K_{2}(p) F_{2}(p \sigma(t))=\Omega_{H},
$$

где $p=d / d t$ - оператор дифференцирования, $\sigma(t)$ - разность фаз эталонного и подстраиваемого генераторов, $\Omega_{1}$ - полоса удержания кольца фазовой автоподстройки частоты (ФАПЧ), $\Omega_{2}$ - полоса удержания частотного кольца, $K_{1}(p)$ и $K_{2}(p)$ - коэффициенты передачи фильтров нижних частот в фазовой и частотных цепях управления, $F_{1}(\sigma(t))$ и $F_{2}(p \sigma(t))$ - характеристики фазового и частотного детекторов, $\Omega_{H}$ - начальная расстройка. Заменой переменных операторное уравнение (1) сводится к системе дифференциальных уравнений, определяющую математическую модель системы частотно-фазовой автоподстройки частоты (см. $[1,5-14])$

$$
\dot{x}=A x+b \varphi(\sigma)+d \frac{2 \alpha \beta c^{T} x}{1+\beta^{2}\left(c^{T} x\right)^{2}}, \quad \dot{\sigma}=c^{T} x,
$$

где $A$ - квадратная $(n \times n)$-матрица, $x, b, c, d \in \mathbb{R}^{n}, \alpha, \beta \in \mathbb{R}, \varphi(\sigma)-\Delta$-периодическая непрерывно дифференцируемая функция. 
Теория систем фазовой синхронизации регулярных сигналов достаточно хорошо развита. В фазовом пространстве системы режиму синхронизации соответствует устойчивое состояние равновесия. Для систем фазовой автоподстройки (ФАП) в работах В. В. Матросова, В. Д. Шалфеева (см. $[1,15,16])$ показано, что при разных видах фильтра нижних частот может произойти нарушение устойчивости состояния равновесия и возникновение около него устойчивого предельного цикла. В этом случае в системе устанавливается квазисинхронный режим, для которого усредненная частота колебаний генератора совпадает с частотой внешнего сигнала, что определяет существование режима фазовой синхронизации. Скрытая синхронизации основывается на понятии «скрытые колебания», сформулированного в работах Г. А. Леонова, Н. В. Кузнецова, И. М. Буркина. Характерной особенностью скрытых колебаний является невозможность попадания на него по траектории с начальными значениями из окрестности состояния равновесия. В данной работе под скрытой синхронизацией понимается наличие в системе устойчивого цикла первого рода, для которого усредненная частота колебаний генератора совпадает с частотой внешнего сигнала, при этом цикл не является «глобально устойчивым».

Определение 1 (см. $[15,16])$. Пусть для системы $(2)$ существует цикл первого рода $z^{*}(t)=$ $\operatorname{colon}\left(x^{*}(t), \sigma(t)\right)$ с периодом $T$, для которого

$$
\langle\dot{\sigma}\rangle=\frac{1}{T} \int_{0}^{T} \dot{\sigma}(t) d t=0 .
$$

Тогда цикл $z^{*}(t)$ определяет квазисинхронный режим системы фазовой синхронизации.

С квазисинхронными режимами связано явление джиттера, которое определяется как отклонение временного положения информационных сигналов в трактах вычислительных и телекоммуникационных устройств от заданных значений. Джиттер является следствием совокупного действия множества дестабилизирующих факторов, специфичных для разных классов устройств хранения и передачи данных. Одним из факторов появления джиттера является фазовая модуляция сигнала; при этом частота отклонения фазы называется частотой джиттера. Проблема выделения компонент джиттера представляет собой сложную техническую задачу, требующую разработку математического аппарата определения фазовой синхронизации.

Определение 2. Пусть у системы (2) существует цикл первого рода $z^{*}(t)$, определяющий режим квазисинхронизма системы фазовой автоподстройки, который не является «глобально» устойчивым. Тогда система ФАП обладает скрытой синхронизацией.

В работе рассматриваются механизмы возникновения скрытой синхронизации; один из таких механизмов связан с использованием частотного управления радиотехнической модели (1). На базе принципа тора (см. [5]) разработаны аналитические методы определения квазисинхронных режимов (см. $[9,10,12,13])$, которые позволяют использовать численные методы анализа частотно-амплитудных характеристик квазисинхронных режимов для системы ЧФАПЧ с фильтрами второго порядка. В $[9,10,12,13]$ предлагаются новые конструкции для построения положительно инвариантных тороидальных множеств, используемых для нахождения начальных условий колебательных циклов системы (2). Примеры таких множеств изображены на рис. 1(а) (множество построено на основе цилиндрических поверхностей второго порядка, без использования решения матричных уравнений) и на рис. 1(b) (множество получено с помощью решения системы трех матричных уравнений, определяющего границы сферической поверхности).

Установлено, что устранение частотного кольца приводит к сохранению квазисинхронных режимов системы ФАПЧ, что позволяет определить численный метод обнаружения скрытой синхронизации системы ФАПЧ при отсутствии частотного кольца (см. [10]). В настоящее время известны многочисленные результаты, связанные с бифуркацией колебательного цикла. Однако малоизученным является вопрос о количестве и методах обнаружения неустойчивых циклов, возникающих в результате бифуркации. Бифуркации циклов приводят к изменению характеристики $\langle\dot{\sigma}\rangle$ квазисинхронного режима. Для оценки качества скрытой синхронизации предлагается использовать мультипликаторы циклов. В системе (2) происходит бифуркация устойчивого 


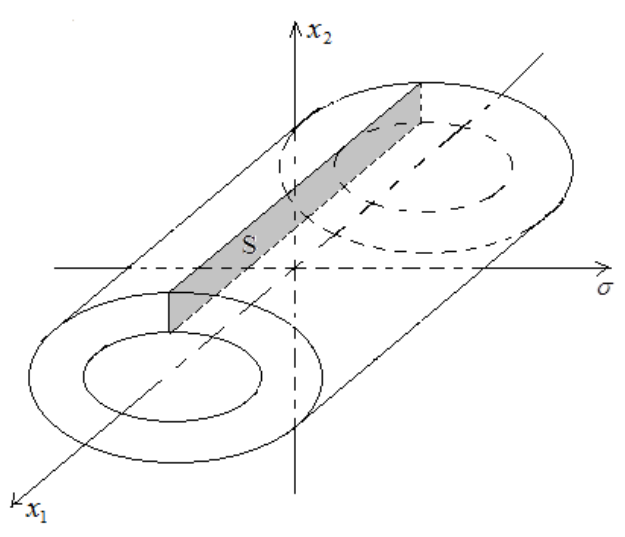

(a)

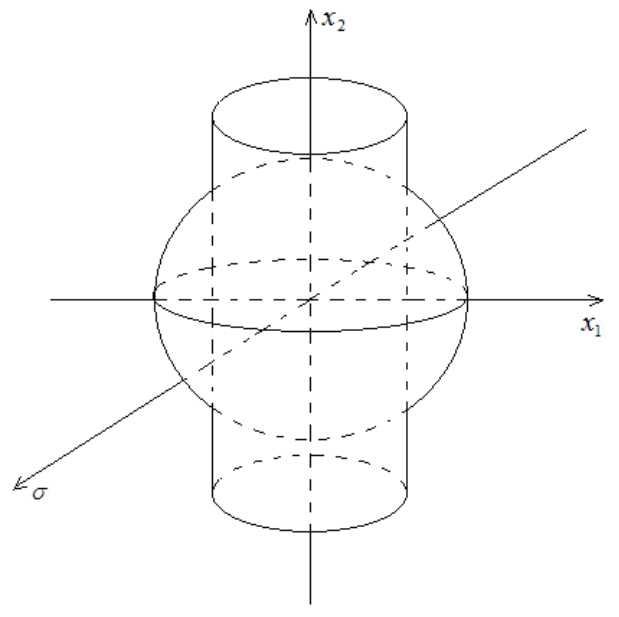

(b)

Рис. 1

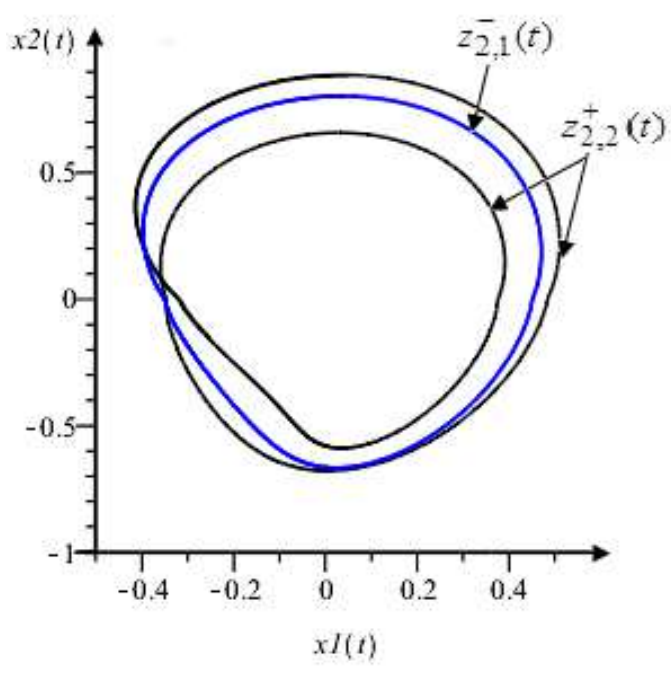

(a)

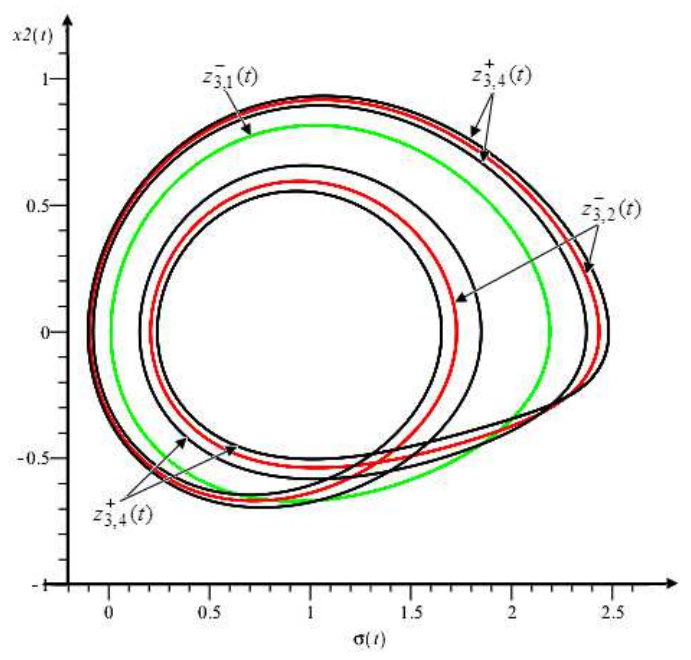

(b)

Рис. 2

однооборотного цикла $z_{1}^{+}(t)$ в устойчивый двухоборотный цикл $z_{2,2}^{+}(t)$; при этом наблюдается неустойчивый однооборотный цикл $z_{2,2}^{-}(t)$ (см. [9]). В дальнейшем появляется один однооборотный неустойчивый цикл $z_{3,1}^{-}(t)$, один двухоборотный неустойчивый цикл $z_{3,2}^{-}(t)$ и один четырехоборотный устойчивый цикл $z_{3,4}^{+}(t)$; при этом неустойчивый цикл $z_{2,1}^{-}(t)$ трансформируется в неустойчивый цикл $z_{3,1}^{-}(t)$. На рис. $2\left(\right.$ a) изображены циклы $z_{2,1}^{-}(t), z_{2,2}^{+}(t)$, а на рис. $2(\mathrm{~b})$ - циклы $z_{3,1}^{-}(t), z_{3,2}^{-}(t), z_{3,4}^{+}(t)$.

Численный анализ показал, что при бифуркации цикла происходит изменение значений

$$
\left\langle\dot{\sigma}_{z_{1}^{+}(t)}\right\rangle=0,008, \quad\left\langle\dot{\sigma}_{z_{2,2}^{+}(t)}\right\rangle=0,0589, \quad\left\langle\dot{\sigma}_{z_{3,4}^{+}(t)}\right\rangle=0,003
$$




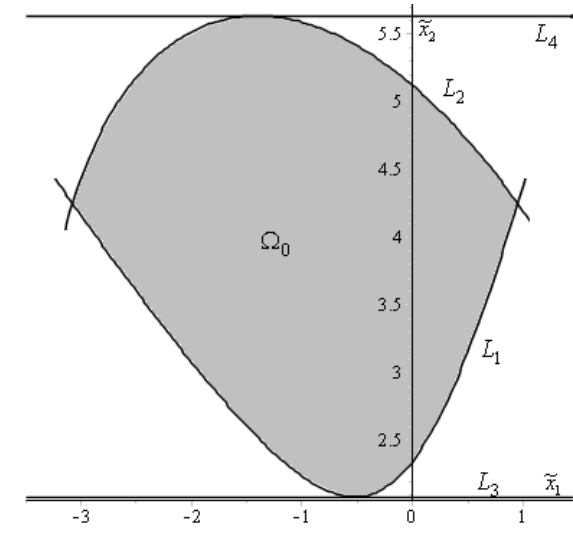

(a)

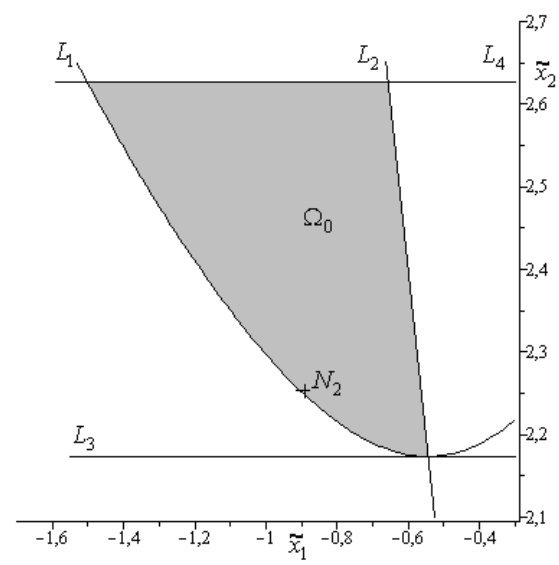

(b)

Рис. 3

для качества синхронизации получены мультипликаторы циклов

$$
\begin{array}{lll}
\mu_{1}\left(z_{1}^{+}(t)\right)=-0,81125, & \mu_{2}\left(z_{1}^{+}(t)\right)=-0,01725, & \mu_{1}\left(z_{2,2}^{+}(t)\right)=-0,14303, \\
\mu_{2}\left(z_{2,2}^{+}(t)\right)=-0,00032, & \mu_{1}\left(z_{3,4}^{+}(t)\right)=0,19763, & \mu_{2}\left(z_{3,4}^{+}(t)\right)=1,7402 \cdot 10^{-7} .
\end{array}
$$

Следующий механизм появления скрытой синхронизации связан с появлением в системе (2) циклов второго рода или вращательных режимов в системе ФАПЧ.

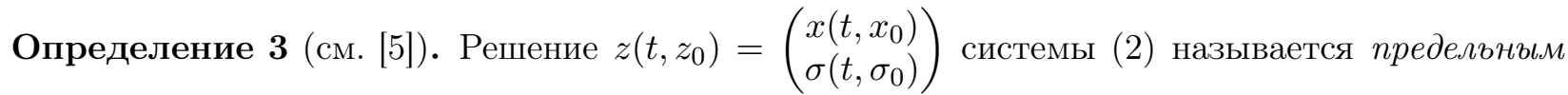
ииклом второго рода или вращательным ииклом, если существуют такие $\tau>0$ и целое число $j \neq 0$, что для любого $t$ выполняются равенства

$$
x\left(t+\tau, x_{0}\right)=x\left(t, x_{0}\right), \quad \sigma\left(t+\tau, \sigma_{0}\right)=\sigma\left(t, \sigma_{0}+\Delta_{j}\right) .
$$

В случае реализации в системе вращательного цикла средняя частота колебаний генератора и частота внешнего сигнала не совпадают, что приводит к появлению у системы асинхронного режима (см. $[2,3,15,16])$. Вращательные режимы предшествуют режиму синхронизации, что позволяет рассматривать их как генератор модулированных колебаний. Реализация в системе нескольких вращательных режимов позволяет выделить в системе область притяжения, которая определяет начальные условия режимов скрытой синхронизации системы ЧФАПЧ. Сечения положительно инвариантных множеств, используемых для нахождения начальных условий устойчивых предельных циклов второго рода системы (2), изображены на рис. 3 (см. [7,8]).

На рис. 4(а) изображены области $\Omega_{10}$ и $\Omega_{20}$, определяющие положительно инвариантные множества, содержащие начальные условия двух предельных циклов второго рода системы (2). На рис. 4(b) изображена область $\Omega_{3}$, определяющая гиперболически инвариантное множество, содержащее начальные условия неустойчивого предельного цикла второго рода (см. [8]).

Система ЧФАПЧ, в зависимости от характеристик частотного кольца, наряду с квазисинхронными режимами может обладать и вращательными режимами. Таким образом, существование в системе ЧФАПЧ одновременно колебательных и вращательных режимов обеспечивает наличие скрытой синхронизации. В случае отсутствия частотного кольца в системе ФАПЧ, множителя $K_{2}(p)$ в уравнении (1) и $\alpha=0$ в системе $(2)$, механизм появления скрытой синхронизации связан с изменением затухания фильтра нижних частот. Если фильтр нижних частот системы ФАПЧ задается равенством

$$
K_{1}(p)=\frac{A_{1} p+A_{2}}{B_{0} p^{2}+B_{1} p+B_{2}},
$$




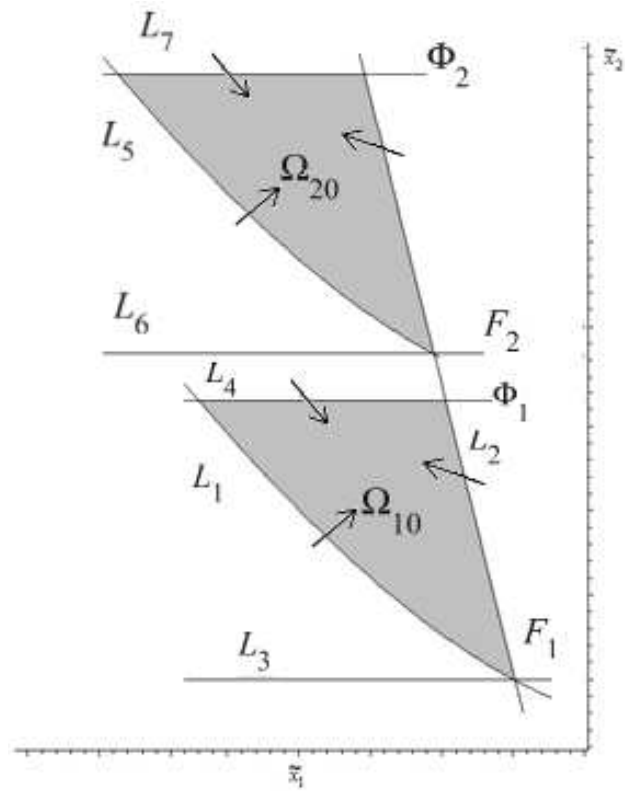

(a)

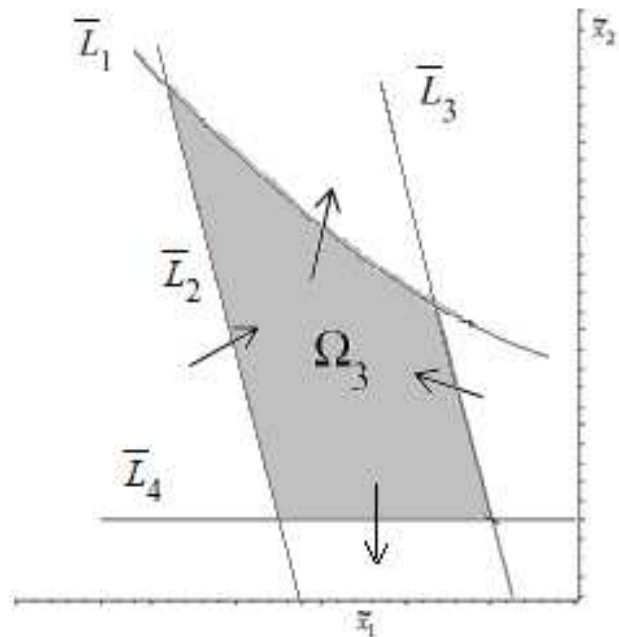

(b)

Рис. 4

то ограниченность затухания системы ФАПЧ определяет знак параметра

$$
\nu=l^{T} b=\left(B_{1} A_{1} B_{0}^{-1}-A_{2}\right) B_{0}^{-1}=-\nu_{1},
$$

где $l^{T}=c^{T} A$.

Для математической модели системы ФАПЧ рассматривается вспомогательная нефазовая система дифференциальных уравнений

$$
\dot{x}=A x+b \varphi(\sigma), \quad \dot{\sigma}=c^{T} x+u \psi(\sigma),
$$

где $x, b, c \in \mathbb{R}^{n}, u \in \mathbb{R}, c^{T} b=-\Gamma<0, c^{T} A=l^{T}, l^{T} b=-\nu_{1}, c^{T} A^{-1} \neq 0$, rank $\|c, l\|=2$, $l^{T} A=-\alpha_{1} l^{T}-\beta_{1} c^{T}, \alpha_{1}>0, \beta_{1}>0, \psi(\sigma)=\nu_{1} \beta_{1}^{-1} \varphi(\sigma)-\alpha_{1} \sigma, \varphi(\sigma)-\Delta$-периодическая функция, имеющая два нуля на периоде $\varphi\left(\sigma_{1}\right)=\varphi\left(\sigma_{2}\right)=0,0=\sigma_{1}<\sigma_{2}<\Delta, \dot{\varphi}(0)>0, \dot{\varphi}\left(\sigma_{2}\right)<0$, производная $\dot{\varphi}(\sigma)$ ограничена на сегменте $[0 ; \Delta]$. Аналитически определяются условия существования циклов первого рода многомерной нефазовой системы дифференциальных уравнений (3) (см. [12]). С использованием пакета прикладных программ Maple разработаны численные методы анализа трансформации цикла нефазовой системы (3) в цикл фазовой системы дифференциальных уравнений (2). Предложены критерии близости циклов двух систем, основанные на метрических характеристиках циклов и мультипликаторном анализе (см. [12]). Для системы (2) проведена классификация сложных колебательно-вращательных режимов системы ФАПЧ, сопровождающих скрытую синхронизацию.

Определение 4. Предельный цикл второго рода $\omega\left(t, z_{0}\right)=$ colon $\left(x\left(t, z_{0}\right), \sigma\left(t, z_{0}\right)\right)$ называется колебательно-вращательным ииклом структуры $\left(k_{1}, k_{2}, \ldots, k_{m}\right)$, если для него существует такое $T>0$, что для любого $t \geqslant 0$ выполняются равенства

$$
x\left(t+T, z_{0}\right)=x\left(t, z_{0}\right), \quad \sigma\left(t+T, z_{0}\right)=\sigma\left(t, z_{0}\right)+m \Delta,
$$

$\Delta$ - период функции $\varphi(\sigma)$ системы $(2)$, функция $\dot{\sigma}(t)=c^{T} x$ имеет $2 k_{i}$ нулей на промежутке $\sigma \in[(i-1) \Delta ; i \Delta], i=\overline{1, m}$. 


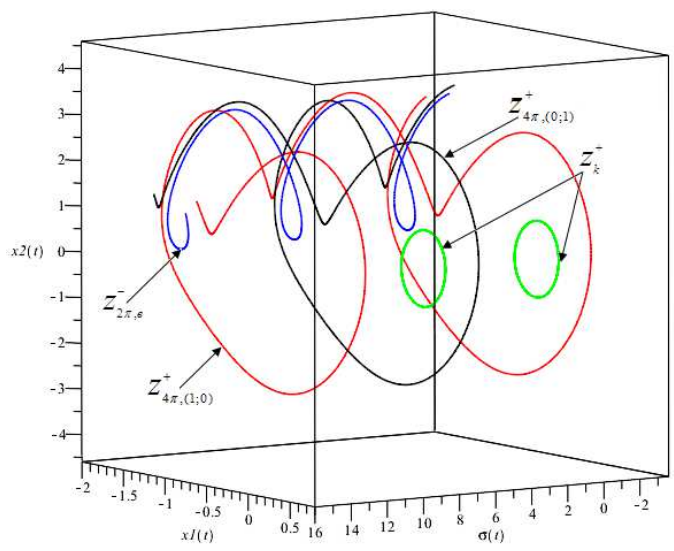

(a)

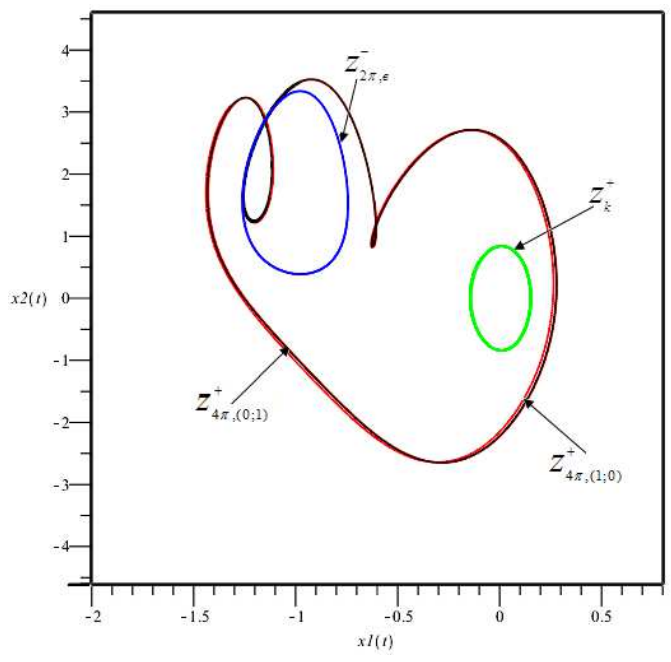

(b)

Рис. 5

В частности, если цикл второго рода $\omega(1,0,2)$ имеет структуру $(1,0,2)$, то для него $m=3$ и существует такое $T>0$, что для любого $t \geqslant 0$ выполняются равенства

$$
x\left(t+T, z_{0}\right)=x\left(t, z_{0}\right), \quad \sigma\left(t+T, z_{0}\right)=\sigma\left(t, x_{0}\right)+6 \pi, \quad \Delta=2 \pi .
$$

Функция $\dot{\sigma}(t)=c^{T} x$ имеет два нуля на промежутке $\sigma \in[0 ; 2 \pi]$, не имеет нулей на сегменте $\sigma \in[2 \pi ; 4 \pi]$ и имеет четыре нуля на промежутке $\sigma \in[4 \pi ; 6 \pi]$. Значения $2 k_{i}, i=\overline{1, m}$, определяют колебательные характеристики цикла $\omega\left(t, z_{0}\right)$, а именно, число оборотов проекции цикла на плоскость $\left(x_{1}, x_{2}\right)$.

У системы (2) наряду с устойчивым колебательным циклом при изменении параметра $\alpha_{1}$ могут появляться как вращательные, так и колебательно-вращательные предельные циклы (см. [14]).

На рис. 5(а) изображены два устойчивых колебательно-вращательных цикла $z_{4 \pi,(1 ; 0)}^{+}(t)$, $z_{4 \pi,(0 ; 1)}^{+}(t)$, неустойчивый цикл второго рода $z_{2 \pi, \mathbf{B}}^{-}(t)$ и устойчивые циклы первого рода $z_{k}^{+}(t)$. На рис. 5(b) изображены проекции циклов $z_{k}^{+}(t), z_{2 \pi, \mathrm{B}}^{-}(t), z_{4 \pi,(1 ; 0)}^{+}(t), z_{4 \pi,(0 ; 1)}^{+}(t)$ на плоскость $\left(x_{1}, x_{2}\right)$; при этом проекции колебательно-вращательных циклов $z_{4 \pi,(1 ; 0)}^{+}(t), z_{4 \pi,(0 ; 1)}^{+}(t)$ на плоскость $\left(x_{1}, x_{2}\right)$ совпадают.

Условиями формирования скрытой синхронизации являются наличие в системе фазовой автоподстройки мультистабильности. Под мультистабильностью понимают сосуществование в фазовом пространстве нескольких аттракторов; в частности, аттракторами могут являтся предельные циклы. Одним из условий появления мультистабильности в системе ФАПЧ является использование запаздывания. Динамика рассматриваемой системы фазовой автоподстройки с запаздыванием описывается операторным уравнением

$$
p \sigma+K_{\varphi}(p) K_{\tau} \Omega_{y} F(\sigma)=\Omega_{H}
$$

(см. [16]), где $K_{\varphi}(p)$ - коэффициент передачи фильтров нижних частот в фазовой цепи управления, $K_{\tau}(p)=e^{-\tau p} \approx(1-\tau p)$ - операторный коэффициент запаздывания, $\tau$ - время запаздывания.

Для математической модели система ФАПЧ с запаздыванием рассматривается вспомогательная система дифференциальных уравнений

$$
\dot{x}=A x+b \varphi(\sigma), \quad \dot{\sigma}=c^{T} x+\rho_{1} \varphi(\sigma)+\rho_{0}(1-u) \varphi(\sigma)-\alpha_{1} u \sigma,
$$

где $x, b, c \in \mathbb{R}^{2}, u \in[0 ; 1], \alpha_{1}, \rho_{1}, \rho_{0} \in \mathbb{R}$. Запаздывание в системе автоподстройки определяет значение параметра $\rho=\rho_{1}+\rho_{0}>0$; при отсутствии запаздывания выполняется равенство $\rho=0$. 
При $u=0$ система (5) примет вид

$$
\dot{x}=A x+b \varphi(\sigma), \quad \dot{\sigma}=c^{T} x+\rho \varphi(\sigma) .
$$

Аналитическими методами определяются условия для параметра $u=u^{*}$, при котором существует цикл многомерной нефазовой системы дифференциальных уравнений (5) (см. [11]). Численными методами определяется трансформация цикла системы (5) в цикл системы (6) при $u=0$. В частности, показано, что система (5) при $u=0,918$ имеет устойчивый цикл первого рода $z_{1}(t)$ с начальными условиями

$$
x_{1}=-0,047922, \quad x_{2}=6,207151, \quad \sigma=0,1, \quad\langle\dot{\sigma}\rangle=0,0044
$$

(см. [11]). Мультипликаторы цикла $z_{1}(t)$ определяются значениями

$$
\mu_{1}=1,0, \quad \mu_{2}=0,102437, \quad \mu_{3}=0,956714 .
$$

Численно показано, что при уменьшении значения $u$ до $u=0$ цикл $z_{1}(t)$ системы $(5)$ трансформируется в цикл $z_{1_{0}}(t)$ фазовой системы $(6)$ с начальными условиями

$$
x_{1}=-0,866482, \quad x_{2}=5,913351, \quad \sigma=0,1, \quad\langle\dot{\sigma}\rangle=0,00061
$$

и мультипликаторами

$$
\mu_{1}=1,0, \quad \mu_{2}=0,1065, \quad \mu_{3}=0,9744 .
$$

При этом мультипликаторный коэффициент аппроксимации циклов $A_{\rho}=2,83 \%$; следовательно, цикл системы (5) является порождающим для цикла системы (6) (см. [11]).

Аналогичным образом можно выяснить, что система (6) имеет устойчивый цикл $z_{2_{0}}(t)$ с начальными условиями и мультипликаторами

$$
\begin{gathered}
x_{1}=-1,2813, \quad x_{2}=14,3064, \quad \sigma=0,1, \quad\langle\dot{\sigma}\rangle=0,0014, \\
\mu_{1}=1,0, \quad \mu_{2}=0,2861, \quad \mu_{3}=0,9857
\end{gathered}
$$

и неустойчивый цикл $z_{0}^{-}(t)$ с начальными условиями и мультипликаторами

$$
\begin{gathered}
x_{1}=-0,339472, \quad x_{2}=9,911372, \quad \sigma=0,1, \quad\langle\dot{\sigma}\rangle=0,0009, \\
\mu_{1}=1,0, \quad \mu_{2}=3,9, \quad \mu_{3}=1,0159
\end{gathered}
$$

(см. [11]). Взаимное расположение циклов $z_{1_{0}}(t), z_{2_{0}}(t), z_{0}^{-}(t)$ фазовой системы $(6)$ в случае запаздывания изображено на рис. 6(a). Для обнаружения неустойчивого цикла предложены численные методы, основанные на использовании вращения векторного поля (см. $[4,7,9,11,12])$. На рис. 6 (b) изображены режим биения при отсуствии затухания $\rho=0$ и квазисинхронные режимы при наличии затухания $\rho=1,16$.

Показано, что механизмом возникновения скрытой синхронизации является формирование мультистабильности, связанной с характеристиками запаздывания. Мультипликаторы циклов являются показателями устойчивости цикла или быстроты стремления траектории к циклу, поэтому предложено использовать мультипликаторы цикла как критерий выбора квазисинхронных режимов в случае мультистабильности. Запаздывание может быть использовано для подавления хаотически модулированнных режимов биения и формирования на их базе квазисинхронных режимов, обеспечивающих фазовую синхронизацию (см. рис. 6(b)). Полученные результаты позволяют получить практические рекомендации для определения параметров фильров второго порядка с запаздыванием, при которых в системе ФАП наблюдается мультистабильность, т.е. одноврменное наличие квазисинхронных режимов с разными частотными характеристиками, что позволяет использовать систему ФАП как генератор многочастотных колебаний. 


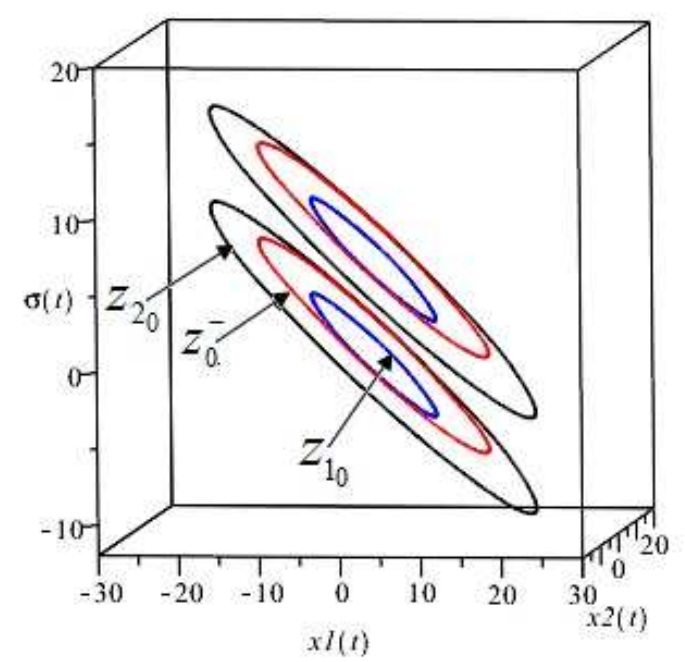

(a)

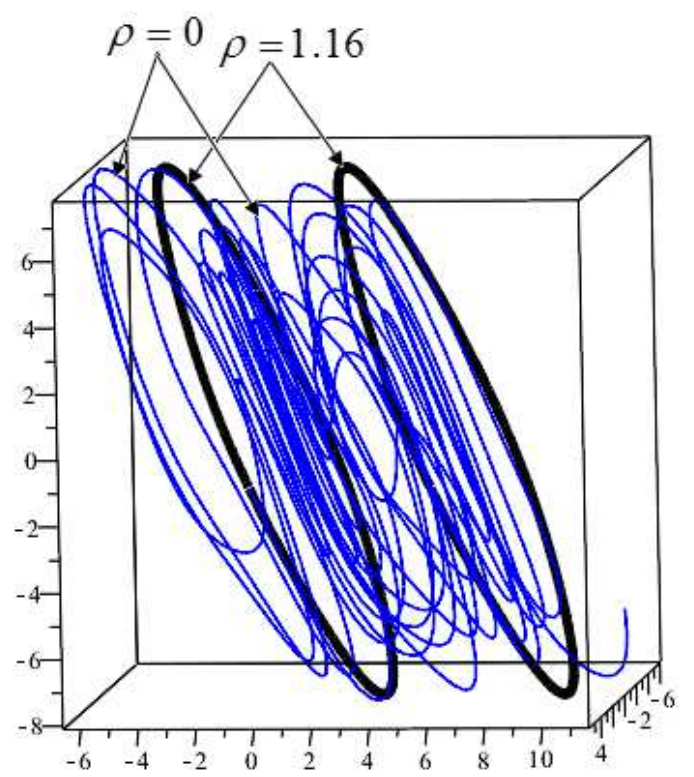

(b)

Рис. 6

\section{СПИСОК ЛИТЕРАТУРЫ}

1. Бакунов Г. М., Матросов В. В., Шалфеев В. Д. О квазисинхронных режимах в системе фазовой автоподстройки частоты с фильтром второго порядка при приближенном учете запаздывания// в кн.: Изв. вузов. Прикл. нелин. динам., 2011. - 19.

2. Бескерский B. А., Попов Е. П. Теория систем автоматического регулирования. - М.: Наука, 1972.

3. Капранов М. В., Кулишов В. Н., Уткин Г. М. Теория колебаний в радиотехнике. - М.: Наука, 1984.

4. Красносельский M. А. Оператор сдвига по траекториям дифференциальных уравнений. - М.: Наука, 1966.

5. Леонов Г. А., Буркин И. М., Шепелявый А. И. Частотные методы в теории колебаний. - СПб.: Изд-во СПбГУ, 1992.

6. Мамонов C. С. Динамика системы частотно-фазовой автоподстройки частоты с фильтрами первого порядка// Вестн. Новосиб. гос. ун-та. Сер. Мат. Мех. Информ. - 2011. - 11, № 1. - С. 70-81.

7. Мамонов С. С., Ионова И. В. Применение вращения векторного поля для определения циклов второго рода// Вестн. РАЕН. Диффер. уравн. - 2014. - 14, № 5. - С. 46-54.

8. Мамонов C. C., Харламова А. О. Отделение циклов второго рода системы частотно-фазовой автоподстройки частоты// Вестн. РАЕН. Диффер. уравн. - 2015. - 15, № 3. - С. 97-102.

9. Мамонов C. С., Харламова А. О. Квазисинхронные режимы фазовой системы// Вестн. Рязан. гос. радиотехн. ун-та. - 2016. - № 56. - С. 45-51.

10. Мамонов C. C., Харламова A. О. Определение условий существоваия предельных циклов первого рода систем с цилиндрическим фазовым пространством// Ж. Средневолж. мат. о-ва. - 2017. - 19, № 1. - С. 67-76.

11. Мамонов C. С., Харламова А. О. Вынужденная синхронизация систем фазовой автоподстройки с запаздыванием// Вестн. Рязан. гос. радиотехн. ун-та. - 2017. - 62. - С. 26-35.

12. Мамонов C. С., Харламова A. О. Численно-аналитическое определение циклов первого рода фазовой системы дифференциальных уравнений// Вестн. РАЕН. Диффер. уравн. - 2017. — 17, № 4. - С. 4856.

13. Мамонов C. С., Харламова А. О. Циклы первого рода систем с цилиндрическим фазовым пространством// Итоги науки и техн. Совр. мат. прилож. Темат. обзоры. - 2018. - 148. - С. 83-92.

14. Мамонов C. С., Харламова А. О., Ионова И. В. Колебательно-вращательные циклы фазовой системы дифференциальных уравнений// Вестн. РАЕН. Диффер. уравн. - 2018. - 18, № 4. - С. 51-57. 
15. Матросов В. В. Вынужденная синхронизация. - Н. Новгород, 2013.

16. Шалфеев В. Д., Матросов В. В. Нелинейная динамика в радиотехнике. - Н. Новгород: Изд-во ННГУ, 2013.

Мамонов Сергей Станиславович

Рязанский государственный университет имени С. А. Есенина

E-mail: s.mamonov@365.rsu.edu.ru

Ионова Ирина Викторовна

Рязанский государственный университет имени С. А. Есенина

E-mail: i.ionova@365.rsu.edu.ru

Харламова Анастасия Олеговна

Рязанский государственный университет имени С. А. Есенина

E-mail: a.harlamova@365.rsu.edu.ru 